\title{
En bloc spondylectomy for primary malignant fibrous histiocytoma of the thoracic spine with aortic involvement: case report
}

\author{
Aravind Somasundaram, BS, ${ }^{1}$ Robert T. Wicks, MD, ${ }^{1}$ Adrian L. Lata, MD, ${ }^{2}$ Shadi A. Qasem, MD, ${ }^{3}$ \\ and Wesley Hsu, MD1
}

Departments of ${ }^{1}$ Neurosurgery, ${ }^{2}$ Cardiothoracic Surgery, and ${ }^{3}$ Pathology, Wake Forest Baptist Medical Center, Winston-Salem, North Carolina

\begin{abstract}
In this article, the authors describe a 48-year-old man who initially presented with progressively worsening back pain. Magnetic resonance imaging revealed a soft-tissue mass involving the T10-11 vertebral bodies with extension anteriorly into the aorta as well as epidural extension without spinal cord compression. A biopsy of the mass showed findings consistent with a malignant fibrous histiocytoma (MFH). A total en bloc spondylectomy with resection and reconstruction of the involved aorta using a vascular graft was performed. The patient received postoperative radiation therapy and is neurologically intact at 18 months postoperatively. To the authors' knowledge, this is the first reported case of a spinal $\mathrm{MFH}$ resection with aortic reconstruction.
\end{abstract}

http://thejns.org/doi/abs/10.3171/2014.9.SPINE14155

KEY WORDS malignant fibrous histiocytoma; thoracic spinal tumor; aortic reconstruction; mediastinal mass; neoadjuvant chemotherapy; oncology

$\mathrm{M}$ ALIGNANT fibrous histiocytoma (MFH) is a rare high-grade soft-tissue sarcoma with a poor prognosis due to a high rate of local recurrence and metastasis. The majority of tumors arise at the knee, proximal femur, and proximal humerus. ${ }^{26}$ Primary MFH of the spine is extremely rare, with only 32 cases reporting a specific type of intervention to manage this malignant lesion since its initial description in $1975.1-3,5,6,8,12-14,16-18,20,21,24-26$

Since the first description of spondylectomy for a giant cell tumor of the lumbar spine in 1966, total spondylectomy has become a viable treatment option for many primary spinal tumors. ${ }^{23,27}$ Tomita and colleagues in 1994 subsequently described a method of total en bloc spondylectomy, which minimized the intratumoral cutting edge to the bilateral pedicles. ${ }^{27}$ This method allowed for en bloc resection of the posterior elements and the vertebral body. Although the involvement of major vascular structures was initially believed to be a contraindication to total en bloc spondylectomy, a case report describing total en bloc resection of a thoracic chondrosarcoma with involvement of the aorta has since been described?

In this paper we present a total en bloc spondylectomy of an $\mathrm{MFH}$ of the posterior mediastinum invading the spine at T10-11 with resection and reconstruction of the involved aorta using a vascular graft. To our knowledge, this is the first published description of a spinal MFH resection with aortic reconstruction.

\section{Case Report}

History and Examination

A 48-year-old man presented to our institution with 18 months of progressive back pain. His medical history was significant for hypertension and prior cigarette use for 20 years. On examination, he had no weakness or sensory deficits, and he was able to walk without assistance. MRI of the thoracic spine at the time of presentation (Fig. 1) revealed a soft-tissue mass involving the T-10 and T-11 vertebral bodies. There was tumor extension anteriorly into the aorta as well as epidural extension without spinal cord compression. A percutaneous CT-guided core biopsy demonstrated a highly pleomorphic spindle-cell neoplasm. The tumor cells featured a highly pleomorphic spindle-cell neoplasm, enlarged hyperchromatic nu- 

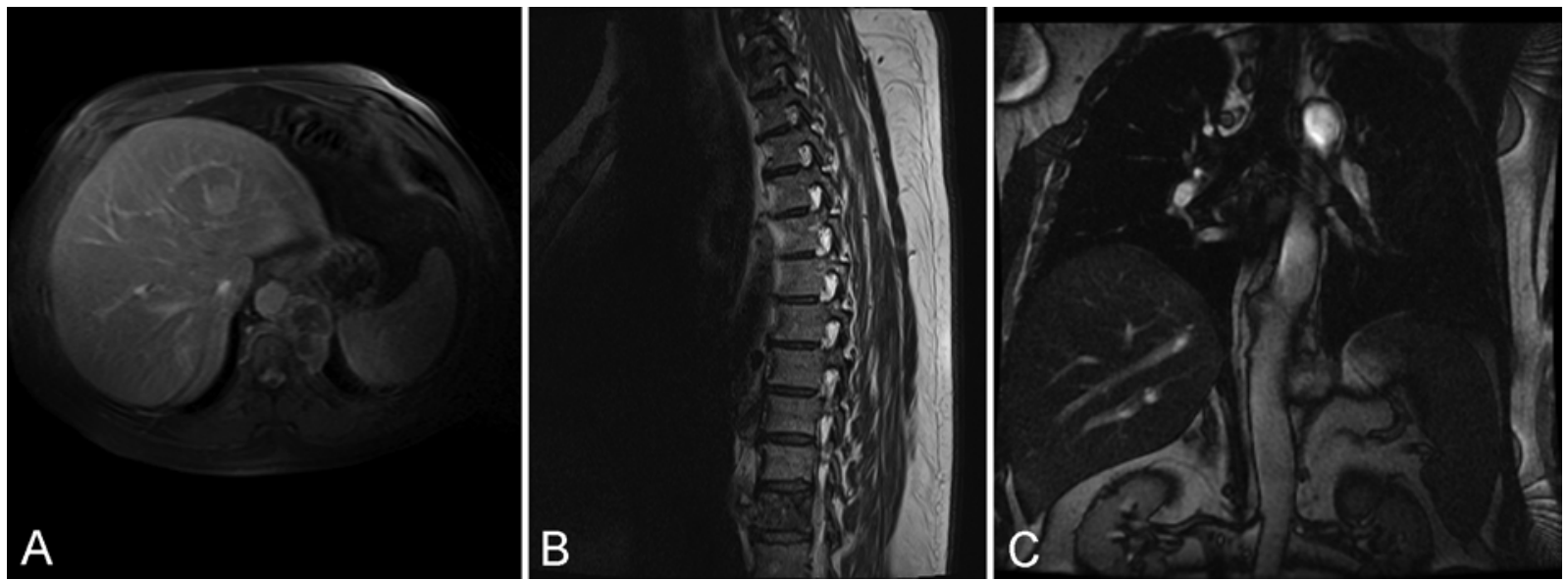

FIG. 1. Preoperative MR imaging of the T10-11 MFH with aortic involvement. A: Axial MR angiogram through T-11 revealing aortic involvement of the MFH tumor and characteristic bone invasion. B: Sagittal T2-weighted MR image revealing T-11 spinal involvement. C: Coronal MR angiogram revealing a periaortic relationship of the MFH tumor.

clei, eosinophilic cytoplasm, and indistinct cytoplasmic borders. Scattered mitoses were noted, but there was no evidence of glandular differentiation (Fig. 2 left). Immunostains showed weak positive staining for smooth muscle actin and negative staining for epithelial and melanocytic markers. These findings were consistent with an MFH.

Given the extent of tumor involvement with the surrounding soft tissue, aorta, and epidural extension, we believed that initial treatment with chemotherapy could lead to a decrease in tumor size and facilitate tumor resection. The patient underwent 3 cycles of neoadjuvant chemotherapy consisting of cyclophosphamide, doxorubicin, and dacarbazine. ${ }^{19}$

MRI performed after chemotherapy demonstrated a decrease in the size of the epidural and paravertebral component of the tumor. We then proceeded with a 2-stage strategy for removing the T10-11 vertebral bodies, residual paravertebral tumor, and involved aorta in an en bloc fashion.

\section{Operative Procedure}

Stage 1. After induction of general anesthesia, the patient was placed prone on a Jackson table. Continuous neuromonitoring was used throughout both stages of the procedure in the form of electromyography, somatosensory evoked potentials (SSEPs), and motor evoked potentials (MEPs). A standard midline exposure of the lamina and lumbar transverse processes from T-7 to L-2 was then performed. We mobilized the paraspinous muscles from the fascia and ribs from T-8 to T-12, thereby allowing us to place rubber catheters around the muscles and retract the muscles medially or laterally. Medial retraction of these muscles improved visualization of the lateral anatomy of the spine. On preoperative imaging the patient was noted to have a 13th thoracic vertebral body. Pedicle screws were placed bilaterally in a standard fashion at T7-9 and T12L2. We performed a laminectomy of T-9 with removal of the bilateral inferior articulating facets, which exposed the superior articulating facets of T-10 along with the exiting T-9 nerve roots. Next, a laminectomy of T-12 with resec- tion of the majority of the bilateral superior articulating facets was conducted. Care was made to disarticulate the T11-12 facet joint, which would be necessary to remove the posterior elements of T-10 and T-11 in an en bloc fashion. We then made osteotomies on the bilateral T-10 and T-11 ribs approximately $3 \mathrm{~cm}$ from the articulation between the rib and transverse processes. We undermined the rib from the parietal pleura and disarticulated the ribs from the vertebral bodies using an osteotome. After medializing the paraspinous muscles, the plane between the thoracic segmental vessels and the lateral aspect of the T-10 and $\mathrm{T}-11$ vertebral bodies was dissected. We first dissected the T-10 and T-11 pedicles. Using a diamond bur, we carefully thinned the pedicle in a lateral to medial trajectory until the medial aspect of the pedicle was amenable to removal with Kerrison rongeurs. We were able to carefully remove the remaining soft tissue and ligamentous attachments to remove the T-10 and T-11 posterior elements en bloc. This allowed us to visualize the exiting nerve roots and thecal sac from T-9 to T-12. The bilateral T-10 and T-11 nerve roots were doubly ligated and sacrificed proximal to the dorsal root ganglion using 2-0 silk ties. The thecal sac was cleanly dissected from the epidural space using a Woodson elevator.

We continued our dissection of the lateral aspect of the vertebral bodies of T-10 and T-11 as well as the T9-10 and T11-12 disc spaces. We followed the plane anteriorly until we were able to palpate the anterior aspect of the tumor. We were able to circumferentially dissect the T11-12 disc space and much of the T-11 vertebral body. Because of the location of the tumor in close proximity to the T9-10 disc space, we were unable to circumferentially dissect the disc space. Using a No. 15 scalpel blade, the posterior and lateral aspects of the T11-12 disc were incised, and the disc was removed using a pituitary rongeur and down-angled curette. Care was made to favor the T-12 superior endplate when removing disc material, as we did not want to violate the T-11 vertebral body. We then contoured a titanium rod connecting the pedicle screws on the left side, as the next steps would lead to disarticulation of the T-11 and T-12 

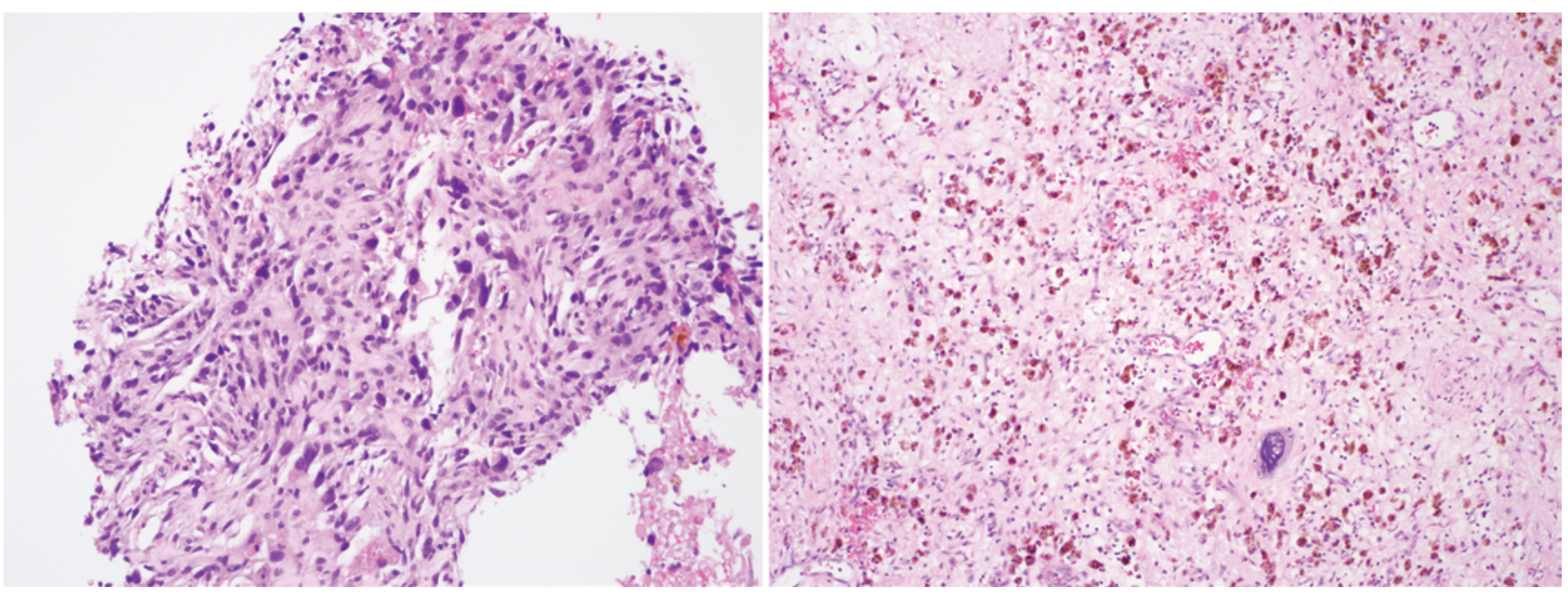

FIG. 2. Photomicrographs of specimens obtained during surgery. Left: Needle biopsy specimen showing a spindle-cell neoplasm with highly pleomorphic nuclei and pink cytoplasm. Right: Resection specimen obtained after adjuvant chemotherapy showing fibrovascular tissue and brown hemosiderin pigment with rare residual tumor cells. H \& E, original magnification $\times 20$ (left) and $\times 10$ (right). Figure is available in color online only.

vertebral bodies. We then passed a 0.81-mm-diameter Tomita saw anterior to the remaining anterior longitudinal ligament at the T11-12 disc level and carefully cut the ligament, thereby completing the disarticulation of the T-11 vertebral body from T-12. Using a No. 15 scalpel blade, the posterior and lateral aspects of the T9-10 disc were incised. Because we were unable to fully dissect the anterior aspect of the disc space at this level, our goal was to remove as much disc material from the inferior endplate of $\mathrm{T}-9$, as the remainder of the disc and anterior longitudinal ligament could be easily incised during the anterior portion of the procedure. DuraGen matrix (Integra, Inc.) was placed between the dura and epidural space and left intentionally for easy identification of the dura during Stage 2 of the operation. We then copiously irrigated the wound and achieved hemostasis. A cutting bur was used to decorticate the remaining lamina and facet joints. Cancellous bone chips and demineralized bone matrix were placed over the remaining posterior elements. We fashioned a rod connecting the right-sided pedicle screw, and both rods were locked in place. We connected the 2 rods using a cross-connecter placed over what was once the T10-11 disc space. Two $10-\mathrm{mm}$ flat drains were placed into the wound and closed in a standard multilayered fashion. The patient was transferred to the neurosciences intensive care unit for overnight observation. The patient had full strength in his lower extremities.

Stage 2. Stage 2 of the procedure was a joint effort among the cardiothoracic and neurosurgical teams. With the patient under general anesthesia, a left-sided doublelumen endotracheal tube was positioned under bronchoscopic guidance. A transesophageal echocardial probe was placed to monitor cardiac activity throughout the case. A Swan-Ganz catheter was placed for monitoring of pulmonary arterial pressure and hemodynamics. A lumbar drain was placed with a goal CSF drainage of $5 \mathrm{ml}$ per hour. Continuous neuromonitoring was again implemented and consisted of electromyography, SSEPs, and MEPs.
The patient was first positioned supine and both groins were prepared. A right femoral arterial line was placed using the Seldinger technique. An oblique incision was then performed over the left groin, and the common femoral artery and vein were exposed. The site was then temporarily closed with staples. The patient was then rotated into the right lateral decubitus position with the upper body positioned at $70^{\circ}$ and the pelvis area at $30^{\circ}$. The left chest wall, abdomen, and groin were prepared and draped. A left posterolateral incision was placed at the seventh rib interspace and carried down to the level of the left upper quadrant of the abdomen. A Tuffier retractor was placed. The costal margin at this level was exposed, and the rectus muscle was divided. The left diaphragm was exposed, and a circumferential incision was performed in the diaphragm extending to the aortic hiatus.

The spleen and left colon were then mobilized toward the midline, and the supraceliac aorta was circumferentially exposed. A piece of umbilical tape was placed around the aorta at this level. Attention was then turned toward the descending thoracic aorta. The lung retracted rostrally and the inferior pulmonary ligament was taken down to the level of the left inferior pulmonary vein. The inferior pulmonary vein was circumferentially exposed. Below the inferior pulmonary vein, the descending thoracic aorta was further exposed. The posterior mediastinal mass invading the spine at T10-11 and invading the descending thoracic aorta at this level was visualized. A harmonic scalpel was then used to divide the parietal pleura and posterior mediastinum, thereby connecting the previous dissection planes created during Stage 1. The aorta proximal and distal to the area of tumor involvement was mobilized from the vertebral bodies, thereby affording access to the T-9 and $\mathrm{T}-12$ vertebral bodies as well as the T9-10 and T11-12 disc spaces.

The T11-12 disc space was identified, and residual soft tissue and disc material were removed from the disc space and the T-12 vertebral body. We then identified the 

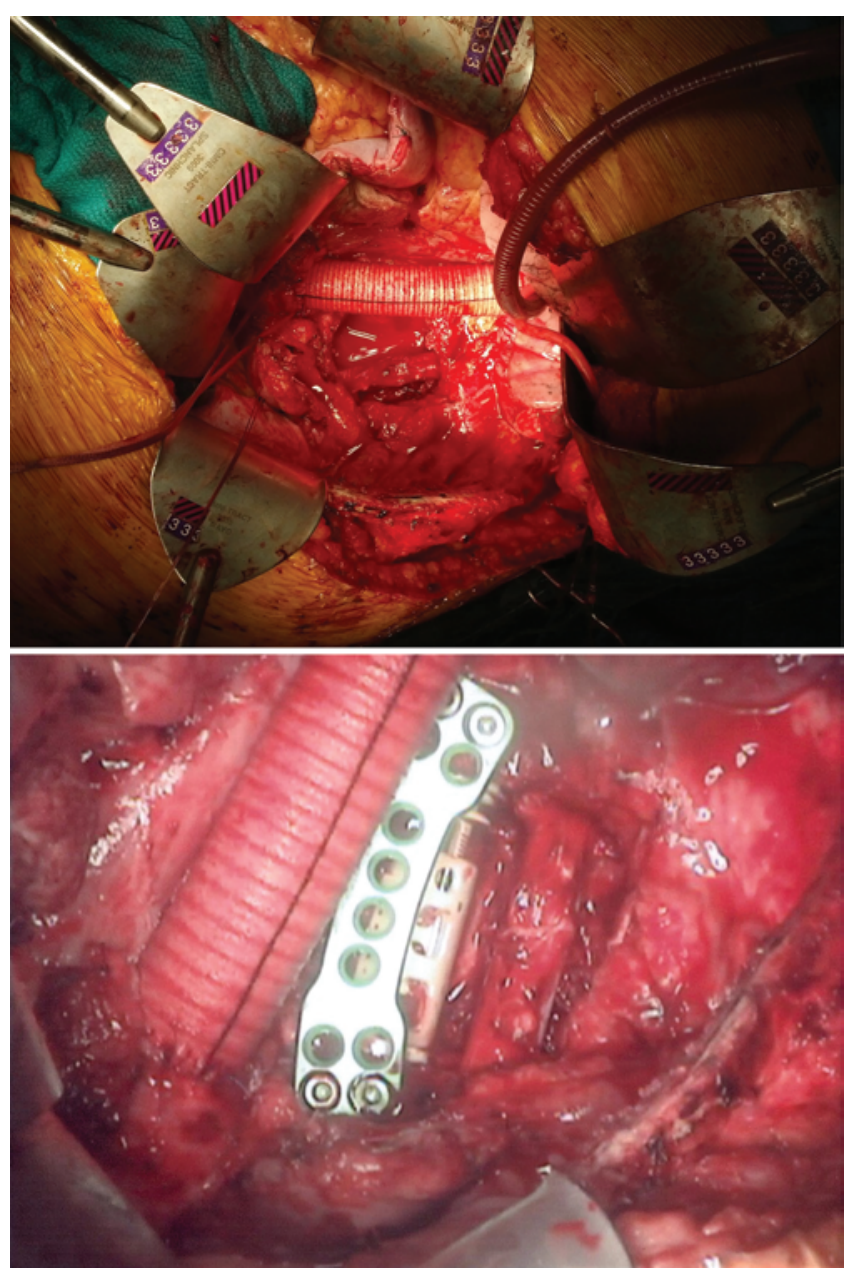

FIG. 3. Intraoperative photographs. Upper: The Hemashield aortic graft prior to discontinuation of left heart bypass. Lower: The Hemashield aortic graft, the plate spanning the T- 9 and T-12 vertebral bodies, and the 4-mm DePuy Spine expandable cage (left to right). Figure is available in color online only.

T9-10 disc space and carefully dissected residual soft tissue away from the disc space and the T-9 vertebral body. The anterior longitudinal ligament and remainder of the disc were excised using a No. 15 scalpel blade and pituitary rongeurs. At this point, the T-10 and T-11 vertebral bodies were disconnected from the remainder of the spinal column. The cardiothoracic surgery team then dissected the exophytic portion of the tumor from the mediastinum to the level of the right pleura.

At this point the patient was systemically heparinized. Via the left groin incision, the left common femoral artery was exposed. A 15-Fr peripheral artery catheter was then positioned into the left common femoral artery under transesophageal echocardiography guidance, and the catheter was secured in place with a Rummel tourniquet. Purse-string sutures were then placed in the left inferior pulmonary vein and a 20 -Fr right-angle venous cannula was placed in the left inferior pulmonary vein toward the left atrium. This, too, was secured with a Rummel tourniquet. Left heart bypass was then established. A trial in-
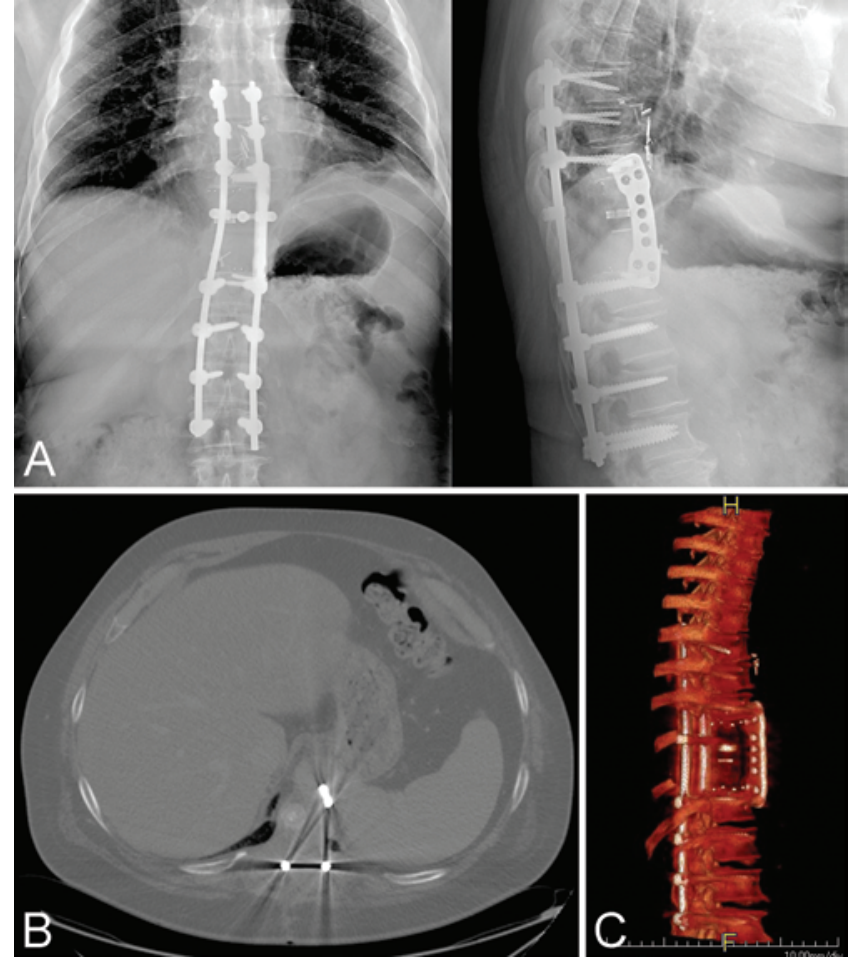

FIG. 4. Postoperative coronal and lateral radiographs (A), axial CT scan (B), and 3D CT scan of the resection space and instrumentation (C). Figure is available in color online only.

volving a cross-clamp on the aorta resulted in no changes to SSEPs.

Proximal and distal control of the aorta was obtained with vascular clamps, and the aorta was completely transected proximally and distally. The T10-11 vertebral body, posterior mediastinal mass, descending thoracic aorta, and periaortic tissue were then mobilized and removed en bloc. Multiple tissue samples from the remaining proximal and distal paraspinal tissue and periaortic tissue were sent for frozen section analysis, and these margin samples were negative for malignancy.

A Hemashield (Atrium Medical Corp.) interposition graft measuring $22 \mathrm{~mm}$ was then used to reconstruct the thoracic aorta. The graft was anastomosed using 4-0 Prolene sutures (Ethicon Endo-Surgery Inc.) in an end-toend nature. The graft was de-aired prior to completion of the anastomosis. After the anastomosis, the graft was filled in an antegrade fashion. Protamine was then given to reverse heparinization, and left heart bypass was discontinued without complication. The left inferior pulmonary vein cannulation site was repaired with 5-0 Prolene sutures.

Once the aortic graft was in place, attention was turned to placing the expandable cage. The vertebral endplates were prepared by removing any remaining cartilaginous material. A 4-mm DePuy Spine expandable cage filled with demineralized bone matrix and cancellous chips was placed in the defect and expanded until resistance from the vertebral bodies was reached. The cage was locked into place. A plate spanning the T-9 and T-12 vertebral bodies was then screwed into place (Figs. 3 and 4). 
Strattice tissue matrix (LifeCell Corp.) was placed between the spinal hardware and the aortic graft. The diaphragm was then repaired with running and horizontal mattress sutures. Bilateral chest tubes were placed within the anterior and posterior left pleural space, and the left lung was inflated. The abdominal wall was then closed using running Prolene No. 1 suture. Ribs were brought together with interrupted Vicryl No. 2 suture (Ethicon Endo-Surgery Inc.). Using running Vicryl suture, the muscle and subcutaneous planes were closed above. The groin site was closed in layers with resorbable sutures. The skin was closed with staples. Examination of the pathology speci- men demonstrated fibrotic tissue with hemosiderin deposition and rare residual tumor cells (Fig. 2 right).

\section{Postoperative Course}

The patient was admitted to the intensive care unit for overnight observation. On postoperative Day 1, he was transferred to a hospital room after removal of the lumbar drain. A CT scan demonstrated excellent position of the cage and pedicle screws with appropriate spinal alignment. He was able to ambulate with a walker and was discharged to a rehabilitation facility on postoperative Day 7. After surgery he completed an additional 4 cycles of

TABLE 1. Cases of MFH in the spinal cord

\begin{tabular}{|c|c|c|c|c|c|}
\hline Authors \& Year & Age (yrs), Sex & Level & Metastasis & Intervention & Outcome \\
\hline Newland et al., 1975 & $74, \mathrm{M}$ & T7-9 & Y & XRT + laminectomy & Died (postop Day 1) \\
\hline \multirow[t]{2}{*}{ Kellet \& Dearnaley, 1976} & $64, \mathrm{M}$ & $\mathrm{L} 1-5$ & Y & Biopsy + XRT & Died (9 mos) \\
\hline & $36, M$ & Lumbar involvement & Y & Biopsy + chemo & Died (12 mos) \\
\hline Dahlin et al., 1977 & $\mathrm{~N} / \mathrm{A}$ & Sacrum & NR & NR & NR \\
\hline Guarnaschelli et al., 1979 & $14, \mathrm{M}$ & $\mathrm{T}-5$ & NR & GTR & Alive (6 mos) \\
\hline Kepes, 1979 & $27, F$ & $\mathrm{~L}-4$ & & STR & Died \\
\hline \multirow[t]{3}{*}{ Teddy \& Esiri, 1979} & $52, \mathrm{M}$ & T10-11 & Y & Biopsy & Died (6 mos) \\
\hline & $45, M$ & $\mathrm{~T}-11$ & NR & Biopsy + XRT & NR \\
\hline & $46, M$ & T3-6 & & Biopsy + XRT & NR \\
\hline Helle et al., 1983 & $42, \mathrm{M}$ & T9-10 & Y & GTR + XRT + chemo & Died (11 mos) \\
\hline Rechtine et al., 1984 & $50, \mathrm{M}$ & $\mathrm{C}-2$ & Y & Biopsy & Died (2 mos) \\
\hline Huvos et al., 1985 & NR & Sacrum & NR & NR & NR \\
\hline Bidwell et al., 1987 & $17, \mathrm{~F}$ & $\mathrm{~L}-5$ & NR & Biopsy & $\begin{array}{l}\text { Died (shortly after di- } \\
\text { agnosis) }\end{array}$ \\
\hline Itoyama et al., 1987 & $65, F$ & $\mathrm{~T} 1-2$ & Suspected & GTR + XRT & Died (2 mos) \\
\hline Sturm et al., 1992 & $27, \mathrm{M}$ & $\mathrm{T}-8$ & NR & Corpectomy + XRT & Alive (16 mos) \\
\hline Maillefert et al., 1997 & $40, F$ & T-12, L-2 & NR & Biopsy + XRT + chemo & Died (15 mos) \\
\hline Karantanas et al., 2000 & $46, F$ & T10-11 & NR & Biopsy + chemo & Alive (15 mos) \\
\hline Sharma et al., 2006 & $66, \mathrm{M}$ & T11-L3 & NR & XRT & Died (13 mos) \\
\hline Akai et al., 2006 & $59, F$ & $\mathrm{C} 0-1$ & $\mathrm{~N}$ & STR + XRT + chemo & Died (28 mos) \\
\hline Ochiai et al., 2009 & $75, \mathrm{~F}$ & $\mathrm{~T} 7-8$ & NR & $\mathrm{GTR}+\mathrm{XRT}$ & Alive (5 mos) \\
\hline \multirow[t]{13}{*}{ Teng et al., 2011} & $42, \mathrm{M}$ & T8-10 & Y & XRT + STR + XRT/chemo & Died (17 mos) \\
\hline & $49, \mathrm{~F}$ & $\mathrm{~T}-11$ & Y & Chemo + EB + chemo & Died (33 mos) \\
\hline & $47, \mathrm{~F}$ & $\mathrm{~T}-12$ & Y & XRT/chemo + STR + XRT/chemo & Died (14 mos) \\
\hline & $60, M$ & L-2, It PVSTM at L2-5 & Y & EB + XRT/Chemo & Died (39 mos) \\
\hline & $53, \mathrm{~F}$ & S1-3 & Y & $\mathrm{XRT} /$ chemo + EB + XRT/chemo & Died (20 mos) \\
\hline & $44, \mathrm{~F}$ & T-12 VB, It PVSTM at T9-12 & Y & Chemo + STR + XRT/chemo & Died (14 mos) \\
\hline & $40, \mathrm{~F}$ & $\mathrm{~T}-10$ & $\mathrm{~N}$ & Chemo + EB + chemo & Alive (60 mos) \\
\hline & $27, \mathrm{M}$ & T-12, L-1 VB, rt PE \& rib & Y & Chemo + STR + XRT/chemo & Died (11 mos) \\
\hline & $59, \mathrm{~F}$ & Lt PVSTM at T12-L2 & $\mathrm{N}$ & Chemo + EB + chemo & Died (25 mos) \\
\hline & $29, M$ & L-3 VB, rt PVSTM at L2-5 & Y & XRT/chemo + STR + XRT/chemo & Died (13 mos) \\
\hline & $45, \mathrm{~F}$ & L-5, PVSTM at L2-5 & Y & Chemo + STR + XRT/chemo & Died (26 mos) \\
\hline & $63, \mathrm{M}$ & S3-5 & $\mathrm{N}$ & Chemo + EB + chemo & Died (23 mos) \\
\hline & $56, M$ & S-1, It S-1 joint, paraspinal mass & Y & Chemo + STR + XRT/chemo & Died (13 mos) \\
\hline Sereno et al., 2013 & $25, \mathrm{~F}$ & T-5 & Y & Surgery + chemo & Alive (3 wks) \\
\hline Present case & $48, M$ & T9-11 & $\mathrm{N}$ & Chemo + EB + XRT/chemo & Alive (14 mos) \\
\hline
\end{tabular}

Chemo = chemotherapy; $\mathrm{EB}=$ en bloc resection; $\mathrm{GTR}$ = gross-total resection; N/A = not assessed; NR = not reported; PE = posterior elements; PVSTM = paravertebral soft-tissue mass; STR = subtotal resection; VB = vertebral body; $\mathrm{XRT}=$ radiation therapy. 
neoadjuvant chemotherapy consisting of cyclophosphamide, doxorubicin, and dacarbazine. He then underwent radiation therapy consisting of $6000 \mathrm{cGy}$ to the T8-12 vertebral bodies 6 months after surgery. On his most recent follow-up (18 months postoperatively), a CT scan demonstrated no evidence of tumor progression. He was able to walk without assistance and did not require opioids for pain control.

\section{Discussion}

Primary MFH of the spine is an extremely rare entity that was first reported in 1975 by Newland et al. ${ }^{17}$ Since its initial description, there have only been 34 cases reported in the literature (Table 1). We report a case of MFH in the thoracic spine with aortic infiltration that was managed with en bloc spondylectomy and aortic resection with graft reconstruction.

MFH of the spine has an unfavorable prognosis. ${ }^{26}$ Detection of MFH in the spine is usually made as the disease has progressed to its later stages, negatively impacting the overall patient outcome. Teng et al. recently published the largest case series of MFH in the spine. ${ }^{26}$ They reported a median survival of 18 months, and 1- and 2-year survival rates of $92.3 \%$ and $38.5 \%$, respectively. These findings compared favorably to previous cases in the literature, which had a median survival of 8.7 months and a 2-year survival rate of $6.7 \%$. Despite these improvements, the authors reported a 5-year survival rate of only $7.7 \%$, highlighting the malignant nature of the disease. The current treatment paradigm for managing MFH of the spine is neoadjuvant chemotherapy and en bloc resection followed by chemotherapy and/or radiotherapy. Our patient followed this protocol and underwent radiotherapy following surgery.

Primary malignant tumors of the spinal cord may be managed successfully through en bloc resection to prevent local growth and metastatic spread. ${ }^{4}$ Teng et al. ${ }^{26}$ showed that patients who underwent en bloc resection for MFH of the spine had a median survival of 25 months, while patients with an intralesional resection had a median survival of 14 months. The goal of en bloc resection is to ultimately achieve negative margins around the tumor. The location of the tumor relative to critical neural and vascular structures plays an important role in determining whether en bloc resection is feasible. One limitation believed to preclude total en bloc resection was large vascular involvement of the tumor. Gösling et al. in 2013 reported the first en bloc spondylectomy of a spinal chondrosarcoma with combined resection of an involved aorta. ${ }^{7}$ We elected to pursue an en bloc resection with aortic resection and reconstruction in a 2-staged procedure.

Neoadjuvant chemotherapy is used to optimize patient outcome by inducing regression of the primary tumor before resection if the tumor is large or in close proximity to critical structures. It also may aid in reducing the chance of metastases, obtaining a clear margin during surgery, and helping to plan a postoperative chemotherapy regimen. In our case, the patient underwent 3 cycles of neoadjuvant chemotherapy prior to surgical intervention and 4 cycles of neoadjuvant chemotherapy after surgical intervention but prior to radiation therapy.
The opportunity for metastatic spread of disease can range from $25 \%$ to $75 \%$ for patients harboring an $\mathrm{MFH}$, with a rate of $53 \%$ in patients with an MFH of the spine..$^{10,26}$ The most common site of manifestation is the lung. Factors that may contribute to metastatic spread include tumor diameter $(>10 \mathrm{~cm})$, in-transit metastases, high rates of microsatellites, and inadequate tumor clearance during initial treatment. In this case we demonstrated the feasibility of an en bloc approach to removing primary spine tumors with aortic involvement. We believe that involvement of the aorta is not an absolute contraindication to considering en bloc resection in carefully selected patient populations.

\section{References}

1. Akai T, Yamamoto K, Iida T, Iizuka H, Nojima T: Malignant fibrous histiocytoma in the craniocervical junction presenting with severe occipitalgia. Brain Tumor Pathol 23:101-105, 2006

2. Bidwell JK, Young JW, Saylor L: Malignant fibrous histiocytoma of the spine: computed tomography appearance and review of the literature. J Comput Tomogr 11:355-358, 1987

3. Capanna R, Bertoni F, Bacchini P, Bacci G, Guerra A, Campanacci M: Malignant fibrous histiocytoma of bone. The experience at the Rizzoli Institute: report of 90 cases. Cancer 54:177-187, 1984

4. Clarke MJ, Hsu W, Suk I, McCarthy E, Black JH III, Sciubba DM, et al: Three-level en bloc spondylectomy for chordoma. Neurosurgery 68 (2 Suppl Operative):325-333, 2011

5. Dahlin DC, Unni KK, Matsuno T: Malignant (fibrous) histiocytoma of bone-fact or fancy? Cancer 39:1508-1516, 1977

6. Feldman F, Norman D: Intra- and extraosseous malignant histiocytoma (malignant fibrous xanthoma). Radiology 104:497-508, 1972

7. Gösling T, Pichlmaier MA, Länger F, Krettek C, Hüfner T: Two-stage multilevel en bloc spondylectomy with resection and replacement of the aorta. Eur Spine J 22 (Suppl 3):S363-S368, 2013

8. Guarnaschelli JJ, Wehry SM, Serratoni FT, Dzenitis AJ: Atypical fibrous histiocytoma of the thoracic spine. Case report. J Neurosurg 51:415-416, 1979

9. Helle TL, Hanbery JW, Becker DH: Meningeal malignant fibrous histiocytoma arising from a thoracolumbar myelomeningocele. Case report. J Neurosurg 58:593-597, 1983

10. Henderson MT, Hollmig ST: Malignant fibrous histiocytoma: changing perceptions and management challenges. J Am Acad Dermatol 67:1335-1341, 2012

11. Huvos AG, Heilweil M, Bretsky SS: The pathology of malignant fibrous histiocytoma of bone. A study of 130 patients. Am J Surg Pathol 9:853-871, 1985

12. Itoyama Y, Fukumura A, Itoh Y, Takamura S, Matsukado Y, Tanimura A: Spinal malignant fibrous histiocytoma producing cord compression-case report. Neurol Med Chir (Tokyo) 27:51-55, 1987

13. Karantanas AH, Hytiroglou P, Zibis AH, Markonis A, Papadimitriou CS: Malignant fibrous histiocytoma of the spine causing spinal neural foramen widening. Comput Med Imaging Graph 24:329-332, 2000

14. Kellett RJ, Dearnaley JN: Malignant fibrous histiocytoma with diffuse spinal nerve involvement. J Clin Pathol 29:910915, 1976

15. Kepes JJ: "Xanthomatous" lesions of the central nervous system: definition, classification, and some recent observations, in Zimmerman H (ed): Progress in Neuropathology. New York: Raven Press, 1979, Vol 4, pp 179-213

16. Maillefert JF, Guy F, Coudert B, Piroth C, Arnould L, Tav- 
ernier C: Multifocal malignant fibrous histiocytoma of the spine. Rev Rhum Engl Ed 64:274-277, 1997

17. Newland RC, Harrison MA, Wright RG: Fibroxanthosarcoma of bone. Pathology 7:203-208, 1975

18. Ochiai H, Kawano H, Shimao Y, Hayashi T, Yamada H: Malignant fibrous histiocytoma of the thoracic spine manifesting as rapidly progressive compressive myelopathy. Neurol Med Chir (Tokyo) 49:438-441, 2009

19. Patel SR, Plager C, Papadopoulos NE, Benjamin RS: Myxoid malignant fibrous histiocytoma: experience with chemotherapy. Am J Clin Oncol 18:528-531, 1995

20. Rechtine GR, Hassan MO, Bohlman HH: Malignant fibrous histiocytoma of the cervical spine. Report of an unusual case and description of light and electron microscopy. Spine (Phila Pa 1976) 9:824-830, 1984

21. Sharma H, Mehdi SA, MacDuff E, Reece AT, Jane MJ, Reid R: Paget sarcoma of the spine: Scottish Bone Tumor Registry experience. Spine (Phila Pa 1976) 31:1344-1350, 2006

22. Sereno M, Merino M, Aguayo C, Hernández S, GutiérrezGutiérrez G, Zambrana Tévar F, et al: A major response to trabectedin in metastatic malignant fibrous histiocytoma of the vertebra: a case report and review of the literature. Tumori 99:e43-e48, 2013

23. Stener B: Total spondylectomy in chondrosarcoma arising from the seventh thoracic vertebra. J Bone Joint Surg Br 53:288-295, 1971

24. Sturm PF, Abramovitz J, Wagner C, Ferguson R, Walker S: Malignant fibrous histiocytoma of the spine. A case report and review of the literature. Spine (Phila Pa 1976) 17:975977, 1992
25. Teddy PJ, Esiri MM: Malignant fibrous histocytoma producing spinal cord compression. J Neurol Neurosurg Psychiatry 42:838-842, 1979

26. Teng H, Xinghai Y, Wei H, Huang Q, Xiao J, Zhang C: Malignant fibrous histiocytoma of the spine: a series of 13 clinical case reports and review of 17 published cases. Spine (Phila Pa 1976) 36:E1453-E1462, 2011

27. Tomita K, Toribatake Y, Kawahara N, Ohnari H, Kose H: Total en bloc spondylectomy and circumspinal decompression for solitary spinal metastasis. Paraplegia 32:36-46, 1994

\section{Author Contributions}

Conception and design: Hsu. Acquisition of data: all authors. Analysis and interpretation of data: Hsu, Somasundaram, Wicks, Qasem. Drafting the article: Hsu, Somasundaram, Wicks.

Critically revising the article: Hsu, Somasundaram, Wicks, Lata. Reviewed submitted version of manuscript: all authors. Approved the final version of the manuscript on behalf of all authors: Hsu. Study supervision: Hsu, Lata, Qasem.

\section{Correspondence}

Wesley Hsu, Department of Neurosurgery and Orthopedic Surgery, Wake Forest Baptist Medical Center, Medical Center Blvd., Winston-Salem, NC 27157-1029. email: whsu@ wakehealth.edu. 